\title{
Effect of Radar-Rainfall Errors on Rainfall-Runoff Modeling
}

\author{
Emad Habib $^{1}$, Ananda V. Aduvala ${ }^{2}$, and Ehab A. Meselhe ${ }^{2}$ \\ ${ }^{1}$ Department of Civil Engineering, University of Louisiana at Lafayette, P.O. Box 42291, \\ Lafayette, LA 70504-2291; Ph (337) 482-0638; Fax (337) 482-0631; email: \\ habib@louisiana.edu \\ ${ }^{2}$ Department of Civil Engineering, University of Louisiana at Lafayette, P.O. Box 42291, \\ Lafayette, LA 70504-2291.
}

\begin{abstract}
Recent years have witnessed significant advances in development of operational radarrainfall products. These products are desirable for several hydrologic applications such as flood forecasting and rainfall-runoff modeling. It is recognized that radar-rainfall estimates are associated with unknown uncertainties. The nature of these uncertainties and their impact on the prediction accuracy of hydrologic models is not fully understood. The present study presents an analysis of uncertainties of operational radar-rainfall products and how they propagate into rainfall-runoff models. The study uses NWS Multi-sensor Precipitation Estimator (MPE) radar-rainfall products over the Goodwin Creek experimental watershed. Surface rainfall observations from a dense rain gauge network in the watershed are used to analyze error characteristics of radar products. MPE radar data are used as input to a fully distributed hydrologic model (GSSHA) to simulate runoff response during 11 storms recorded in 2002. The study focuses on effect of three different radar error characteristics: systematic error (bias), random error, and temporal and spatial correlations of radar the error filed.
\end{abstract}

\section{Introduction}

Since the advent of the weather radar systems, numerous studies have been conducted to examine the utility of radar-rainfall information in runoff modeling (Ogden and Julien, 1993; Winchell et al., 1998; Vieux and Bedient, 1998; Borga 2002; and Neary and Habib, 2004). Some of these studies focused on operational aspects of using radar data for flood predictions, while others performed sensitivity analyses using radar data, either synthetic or actual estimates, to examine effects of individual sources of the radar error such as reflectivity-rainrate conversions and variability. A recent study by Hossain et al., (2004) focused on the interaction of model parametric uncertainty with radar errors that are related to variability of reflectivity with height. Other studies relied on using synthetic data to examine radar errors and their propagation (Sharif et al., 2002).

Common to the above mentioned studies is their focus on selected aspects of the radar error. However, in practical applications, separating and estimating the different sources of radar errors is not possible. An alternative approach to this problem deals with time-space stochastic modeling of the combined sources of the radar error (e.g., Krajewski et al., 1993). However, due to lack of accurate surface rainfall measurements, recent applications of this approach in hydrologic modeling studies were based on simplified assumptions regarding the distribution of the radar error and its spatial and temporal dependence properties (e.g., Carpenter and Georgakakos 2004; Butts et al., 
2004). In most error-modeling studies, the spatial and temporal correlations of radar error fields were neglected. The need to account for error dependency was recently investigated (Hossain and Anagnostou, 2006; Nijssen and Lettenmaier, 2004) for satellite precipitation. Jordan et al., (2003) developed a random cascade model of radar errors that accounts for error correlations. However, their model dealt with correlations of reflectivity errors that are caused by variations in vertical reflectivity fields.

In the present study, we follow an approach proposed by Krajewski and Ciach (2004) to examine the marginal and joint statistical properties of the radar error and investigate their relative significance for estimating uncertainties in runoff predictions. Instead of focusing on a certain source of the error, we deal with the combined radar error, which can be estimated by analysis of radar-rainfall data against accurate reference surface rainfall information.

\section{Study Site Description and Rainfall-Runoff Model}

The Goodwin Creek (GC) experimental watershed $\left(21.4 \mathrm{~km}^{2}\right)$ located in north Mississippi was selected for this study (Figure 1). Rainfall and runoff in the watershed is monitored by more than 30 rain gauges and several discharge gauges. In this study, a total of 12 significant rainfall-runoff events during the wet non-growing season are considered (Table 1). These events were grouped into two continuous simulation periods (January-February, and March-May, 2002), to be modeled in a continuous mode. For most of the selected storms, runoff-rainfall ratios were considerably high (up to 86\%), and discharge peaks reached as high as $57.5 \mathrm{~m}^{3} \mathrm{~s}^{-1}$ (equivalent to a unit peak of $2.69 \mathrm{~m}^{3} \mathrm{~s}^{-}$ ${ }^{1} \mathrm{~km}^{-2}$ ).

The MPE operational precipitation products of the NEXRAD system are used in this study. MPE products are hourly accumulations of precipitation over a grid of approximately $4 \times 4 \mathrm{~km}^{2}$ that is usually referred to as HRAP grid. An assessment of the quality and accuracy of operational rainfall estimates is provided in several studies (e.g., Young et al. 2000), which indicated that despite bias adjustment, biases as well as significant random errors are still present in these estimates.

In the current study, the Gridded Surface Subsurface Hydrologic Analysis (GSSHA) model is used to develop a rainfall-runoff model for the GC watershed. GSSHA is a fully distributed-parameter process-based hydrologic model (Downer and Ogden, 2002). The model setup adopted in this study is similar to that used by Senarth et al., (2000) with the following modeling options: two-dimensional diffusive wave for overland flow, one-dimensional explicit diffusive wave method for channel flow, Penman-Monteith equation for evapotranspiration calculations, and the Green and Ampt infiltration with redistribution method for flow simulation in the unsaturated zone. This infiltration method includes soil moisture accounting and allows for a continuous longterm simulation of the selected periods that contain multiple runoff events. Prior to being used to assess the effects of radar-rainfall errors, the GSSHA model was subject to a careful calibration process. The results shown in Figure 2 indicate that the model is well calibrated and will likely provide physically reasonable representation of the important runoff processes within the GC watershed. 


\section{Analysis of Systematic Component of Radar Error}

The most basic characteristic of the radar error is overall bias $(B)$, which represents systematic average deviations of radar estimates $\left(R_{r}\right)$ with respect to the corresponding surface rainfall over the radar pixel size $\left(R_{s}\right)$ :

$B=\frac{E\left[R_{s}\right]}{E\left[R_{r}\right]}$

where $\mathrm{E}[\bullet]$ denotes the expectation operator.

We estimate the bias factors for each radar pixel over the watershed (Figure 1) and for each storm separately (Figure 3). In this formulation, surface pixel-average rainfall rate is obtained by averaging observations from several rain gauges located within the pixel. Although originally adjusted with rain gauge measurements, MPE data still contain considerable bias values that range from about 0.4 to 1.6. As expected, bias varies significantly from one storm to another. Spatial variations in bias estimates across the different pixels are also evident, but to a lesser degree.

Besides the overall bias, Ciach et al., (1999) showed that radar bias can also depend on the magnitude of the estimated rainfall itself. This property, which is termed as "Conditional Bias $(C B)$," describes how a specific radar estimate differs on average from the corresponding surface rainfall rate:

$C B\left(r_{r}\right)=\frac{E\left\{R_{s} \mid R_{r}=r_{r}\right\}}{r_{r}}$

We estimated $C B$ for each storm separately. As expected, CB depends on the radar estimate; however, the behavior of such dependence was different from one storm to another. For most storms (e.g., storm 1) CB decreased with $R_{r}$, which indicates that radar tends to overestimate high rainfall rates and underestimate low rainfall rates. Other storms showed either an opposite behavior (e.g., storm 9), or a rather uniform conditional bias (e.g., storm 12) that does not depend on rainfall intensity.

\section{Effect of Radar Bias on Runoff Prediction}

To investigate the effect of radar bias on rainfall-runoff simulations, we applied three simple correction methods which examine different aspects of radar bias. The first method corrects the overall bias for each storm and ignores bias spatial variations. A second method corrects the bias at each pixel and for each storm. The third method estimates the bias with conditioning on the radar estimate. In this method bias spatial variations could not be considered to avoid further reductions in the sample sizes. For brevity, we will term the three methods as: spatially-uniform unconditional; spatiallydependent unconditional; and spatially-uniform conditional.

The comparison is quantified for each storm in terms of two statistical measures: the ratio of radar to surface rainfall storm totals, and the root mean squares of differences (RMSD) between radar and surface hourly rainfall rates. An example of the comparison results is shown in Figure 4 for the central pixel. The bias correction not only resulted in adjusting storm rainfall totals, but also improved the overall agreement between hourly radar and surface estimates. Compared to the unadjusted MPE data, considerable 
reductions in the RMSD are obtained after applying the bias-correction methods. The most basic bias adjustment (spatially-uniform, unconditional) reduced the radar RMSD in 7 out of the 12 storms. Using conditional-bias adjustment resulted in the most significant reduction in RMSD. Improvements in radar estimates in some storms (e.g., 2, 5, and 6) were obtained only with conditional-bias adjustment.

To examine the effects of different bias characteristics, GSSHA model simulations were driven with several rainfall inputs: original MPE data and MPE data adjusted using each of the three methods described above. We compared the radar-driven simulated hydrographs against "reference" runoff simulations driven by pixel-averaged rain gauge measurements. Differences between radar-driven and reference hydrographs are evaluated for each storm both visually (Figure 5) and statistically (Figure 6). Correction of overall biases has resulted in a significant improvement in the radar-based simulated hydrographs. Runoff bias and RMSD decreased significantly for all of the storms. While most of the simulated hydrographs gained an immediate benefit after removing the overall bias, the next most significant improvement was obtained as a result of accounting for bias dependence on the estimated radar rain rates. While the removal of radar biases resulted in improving the runoff prediction accuracy, residual differences are still apparent in some of the simulated hydrographs. This is probably due to the random radar error component that remains in the radar rainfall input.

\section{Modeling of the Radar Random Error}

After removing the overall and conditional bias the radar error can now be considered purely random, which can be characterized using the following form: $\varepsilon=\frac{R_{s}}{R_{r}}$

Preliminary analysis using variance reduction factors (not shown) indicated that the dense gauge arrangement within the central pixel and two more pixels (upper and middle right pixels) can provide relatively accurate approximation $R_{s}$. Using hourly gauge averages in each of the three selected radar pixels, we computed the radar random error using equation (9). We also computed the empirical cumulative probability distribution of the error and compared it to the cases of normal and lognormal distributions. The lognormal distribution provided a better fit to the random component of the radar error. Due to the applied conditional bias removal, the mean of $\varepsilon$ does not depend on the radar estimate and is almost equal to one. Unlike the mean, the standard deviation of $\varepsilon$ depends on $R_{r}$ and was approximated using the following formula:

$\sigma_{\varepsilon \mid R_{r}}^{2}=\alpha\left(R_{r}\right)^{-\beta}$

\section{Spatial and Temporal Dependence of Radar Error}

We now examine the joint properties of the error distribution to assess its spatial and temporal dependence. We do so by estimating the temporal and spatial autocorrelation of $\varepsilon$. Using the marginal samples of $\varepsilon$ estimated for each of the three selected pixels, we calculated the auto correlation of $\varepsilon$ at different time lags (not shown). We also calculated spatial correlations between errors of the three pairs of pixels combined 
together (Figure 7), which represents the 4-km lag correlation of $\varepsilon$. Results for storms with sample sizes that are too small to be adequate for estimating correlation coefficients are not displayed. The main observation is that both the temporal and spatial correlations of $\varepsilon$ are non-negligible. However, the temporal auto-correlation is rather low (mostly less than 0.4 ) at small lags ( 1 or 2 hours) and becomes close to zero for larger time lags. The spatial correlation exhibits stronger levels for most of the analyzed storms. For some storms, the spatial correlation was high (0.8 and above). When combining all storms, the sampling effect becomes less important and the temporal correlations show more stable, but still low, values. Similarly, the spatial correlations between pairs of pixels, but without accounting for intra-storm variability (solid line in Figure 7), maintains rather medium correlation values $(\sim 0.5)$.

\section{Sensitivity of Runoff Hydrographs}

Using the identified lognormal distribution of the radar error, we generated spatially correlated random fields of $\varepsilon$, which were then substituted in equation (9) to provide the corresponding realizations of probable surface rainfall fields that take into account the identified characteristics of the radar error. Since temporal correlations were found to be rather low, we focused our analysis on accounting for the spatial dependence only. An example of such analysis is shown in Figure (8). Each realization is then used as input to the GSSHA hydrologic model resulting in an ensemble of predicted runoff hydrographs (Figure 8). For most hours within the storm, the reference rainfall input falls within the generated rainfall ensemble. The predicted ensemble runoff hydrographs also enclosed the reference hydrograph that was based on accurate surface rainfall information. This indicates the ability of the fitted-error model to characterize radar uncertainties and their propagation into the hydrologic model, and to explain the observed deviations of the radar-based runoff predictions from the reference hydrographs.

To examine the sensitivity of the propagation of radar uncertainties to different levels of error correlations, we repeated the above analysis using two more correlation values: 0 and 0.95 . The hydrograph ensembles obtained with the three examined spatial auto-correlation levels are summarized in terms of their 2.5 and 97.5 percentiles (Figure 9). The difference between the two percentile lines represents the $95 \%$ uncertainty range in the predicted hydrographs due to the effect of radar random errors. The estimated bounds show clear variations during the storm duration. In general, they are wider during the rising limbs of the hydrographs than during the falling limbs. For storms with singlepeak hydrographs, runoff uncertainty bounds have a well-defined and systematic behavior. They increase gradually along the rising part of the hydrograph until they reach a maximum value just before the hydrograph peaking time, and then start to decrease gradually during the receding part of the hydrograph. For storms with more complex patterns (i.e., with multiple bursts of rainfall and runoff peaks), the patterns and relative magnitudes of the uncertainty bounds become more complex too. The increased runoff uncertainty during the rising parts of the hydrographs reflects the enhanced sensitivity of the early-generated runoff to rainfall variability, and more importantly to uncertainties in the rainfall estimates and the introduced degree of spatial error dependence. 
Using a high error correlation value (0.95) resulted in much wider runoff uncertainty bounds than those obtained with low (0) or medium (0.6) correlations. Again, the runoff uncertainty bounds are more sensitive to error correlations during the rising limbs of the hydrographs, but much less sensitive during falling limbs where the percentile lines of the three spatial correlation levels are almost indistinguishable. It is also worth noting that the degree of sensitivity to the assumed level of error correlation is not always the same as it varies from one storm to another.

\section{Summary and Conclusions}

In this paper we analyzed errors of radar-rainfall estimates focusing on its two main components: systematic (bias) and random. The results indicated that, despite being subject to operational bias-removal procedures, the analyzed MPE radar data still suffered from systematic deviations from the reference surface rainfall. Further adjustments of such deviations seem to provide the most significant improvement in runoff predictions in terms of runoff volumes, coefficient of efficiency, and peak prediction. The overall radar bias showed significant variations both spatially (pixel-topixel) and temporally (storm-to-storm). However, given the limited size of the watershed, accounting for temporal (storm-to-storm) variability proved more important for runoff prediction purposes. Besides the overall bias, further improvements were obtained after correcting for the radar bias in a conditional way.

Radar random errors were estimated empirically based on comparison to rain gauge measurements in three pixels covering the watershed. An empirical model based on the log-normal distribution was fitted to approximate the multiplicative radar random error component. The fitted lognormal distribution has a standard deviation that decreases with the increase of the radar rainfall estimate. We also examined the temporal and spatial dependence of the radar error. One-hour lag temporal correlations were found to be non-negligible, but rather low. The 4-km lag spatial auto-correlation coefficients attained more significant levels for many of the analyzed storms.

Runoff uncertainty bounds caused by the modeled radar uncertainties were estimated and found to be quite wide especially during the rising periods of the hydrographs. These bounds were also sensitive to the assumed error spatial correlation. The sensitivity was most significant during the rising parts of the hydrographs and much less significant during the receding periods. In some of the simulated events, reference runoff simulations were enclosed within the runoff uncertainty bounds only when a certain error correlation level was used to model the radar error spatial dependence. This finding indicates the need for further research aiming towards better characterization of the dependence structure of radar errors and accounting for their effects in radar-based runoff modeling studies.

The complexity evident in the estimated runoff uncertainty bounds calls for more investigations on the propagation of radar errors into runoff predictions. Currently, most studies have focused on analysis of the end predictable variable of these models (streamflow). We believe that tracking the errors through the different simulated hydrologic processes (e.g., infiltration, soil moisture variations, evapotranspiration losses, and surface and channel flow) might give better insight into the error propagation and 
how it interacts with the different modeling components. Current efforts are underway by the authors to implement this process-based error propagation analysis approach.

\section{References}

Borga, M. (2002). "Accuracy of radar rainfall estimates for stream flow simulation." $J$. Hydrol., 267, 26-39.

Butts, M. B., Payne, J. T., Kristensen, M., and Madsen, H. (2004). "An evaluation of the impact of model structure on hydrological modeling uncertainty for streamflow simulation." J. Hydrol., 298, 242-266.

Carpenter, T. M., and Georgakakos, K. P. (2004). "Impacts of parametric and radar rainfall uncertainty on the ensemble streamflow simulations of a distributed hydrological model." J. Hydrol., 298, 202-221

Ciach, G. J., and Krajewski, W. F. (1999). "On the Estimation of radar rainfall error variance." Advances in Water Resources., 22(6), 585-595.

Downer, C. W., and Ogden, F. L. (2002). "GSSHA User's Manual, Gridded Surface Subsurface Hydrologic Analysis Version 1.43 for WMS 6.1." Engineer Research and Development Center Technical Report, Vicksburg, MS.

Hossain, F., Anagnostou, E. N., Borga, M., and Dinku, T. (2004). "Hydrological Model Sensitivity to Parameter and Radar Rainfall Estimation Uncertainty." Hydrological Processes, 18(17), 3277-3299.

Hossain, F., and Anagnostou, E. N. (2006). "A two dimensional satellite rainfall error model." IEEE transactions on geoscience and remote sensing. 44, doi: 10.1109/TGRS.2005.863866).

Jordan P. W., Seed, A. W., and Weinmann, P. E. (2003). "A stochastic model of radar measurement errors in rainfall accumulations at catchment scale." J. Appl. Meteor., 4, 841-855.

Krajewski, W. F., and Ciach, G. J. (2004). "Towards Operational Probabilistic Quantitative Precipitation Estimation Using Weather Radar." 6th Inter. Symposium on Hydrological Applications of Weather Radar, Melbourne, 2-4 February.

Krajewski, W. F., Raghavan, R., and Chandrasekar, V. (1993). "Physically based simulation of radar rainfall data using a space-time rainfall model." J. Appl. Meteor., 32, 268-283.

Neary V., Habib, E., and Fleming, M. (2004). "Hydrologic Modeling with NEXRAD Precipitation." J. Hydrolog. Eng., 9(5), 339-349.

Nijssen, B., and Lettenmaier, D. P. (2004). "Effect of precipitation sampling error on simulated hydrological fluxes and states: Anticipating the Global Precipitation Measurement satellites." J. Geophys. Res.-Atmos., 109, Art. No. D02103, 0.1029/2003JD003497.

Ogden, F. L., and Julien, P. Y. (1993). "Runoff sensitivity to temporal and spatial rainfall variability at runoff plane and small basin scales." Water Resour. Res., 29(8), 25892597.

Senarath, S. U. S., Ogden, F. L., Downer, C. W., and Sharif, H. O. (2000). "On the calibration and verification of distributed, physically-based, continuous, Hortonian hydrologic models." Water Resour. Res., 36, 1495-1510.

Sharif H. O., Ogden, F. L., Krajewski, W. F., and Xue, M. (2002). "Numerical simulations of radar rainfall error propagation." Water Resour., 38(8), 1140. 
Vieux, B. E., and Bedient, P. B. (1998). "Estimation of Rainfall for Flood Prediction from WRS-88D Reflectivity: A Case Study." Weather and Forecasting, 13(2), 407415.

Winchell, M., Gupta, H. V., and Sorooshian, S. (1998). "On the simulation of infiltrationand saturation- excess runoff using radar-based rainfall estimates: Effects of algorithm uncertainty and pixel aggregation." Water Resour. Res., 34, 2655-2670.

Young, C. B., Bradley, A., Krajewski, W. F., Kruger, A., and Morrissey, M. L. (2000). "Evaluating NEXRAD multisensor precipitation estimates for operational hydrologic forecasting." J. Hydrometeorol., 1, 241-254.

Table 1 Summary of rainfall-runoff simulation events.

\begin{tabular}{cccccc}
\hline Storm & $\begin{array}{c}\text { Storm } \\
\text { Date }\end{array}$ & $\begin{array}{c}\text { Storm } \\
\text { duration } \\
\text { (hours) }\end{array}$ & $\begin{array}{c}\text { Total } \\
\text { rainfall } \\
\text { depth }(\mathrm{mm})\end{array}$ & $\begin{array}{c}\text { Total runoff } \\
\text { depth }(\mathrm{mm})\end{array}$ & $\begin{array}{c}\text { Max runoff } \\
\text { peak } \\
\left(\mathrm{m}^{3} / \mathrm{s}\right)\end{array}$ \\
\hline 1 & $1 / 18 / 02$ & 19 & 41.9 & 26.1 & 27.0 \\
2 & $1 / 22 / 02$ & 27 & 36.0 & 23.2 & 25.4 \\
3 & $1 / 24 / 02$ & 26 & 81.5 & 70.6 & 40.6 \\
4 & $2 / 5 / 02$ & 39 & 24.6 & 10.1 & 5.8 \\
5 & $2 / 19 / 02$ & 8 & 44.2 & 25.2 & 36.5 \\
6 & $3 / 15 / 02$ & 11 & 15.7 & 8.8 & 5.8 \\
7 & $3 / 16 / 02$ & 44 & 33.3 & 21.2 & 13.8 \\
8 & $3 / 20 / 02$ & 24 & 38.1 & 27.2 & 17.7 \\
9 & $3 / 26 / 02$ & 9 & 20.3 & 6.3 & 7.0 \\
10 & $3 / 29 / 02$ & 42 & 71.4 & 47.9 & 26.6 \\
11 & $4 / 8 / 02$ & 20 & 24.1 & 4.9 & 6.3 \\
12 & $5 / 2 / 02$ & 61 & 130.6 & 74.3 & 57.5 \\
\hline
\end{tabular}

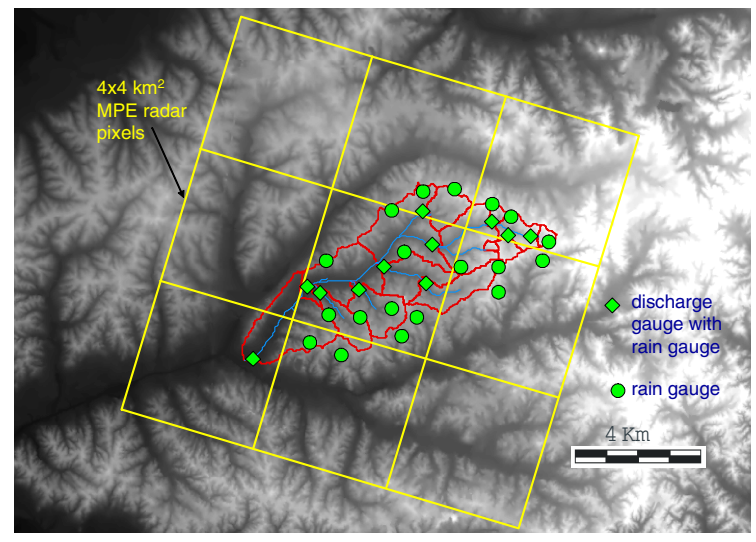

Fig. 1 The Goodwin Creek watershed in north central Mississippi. 

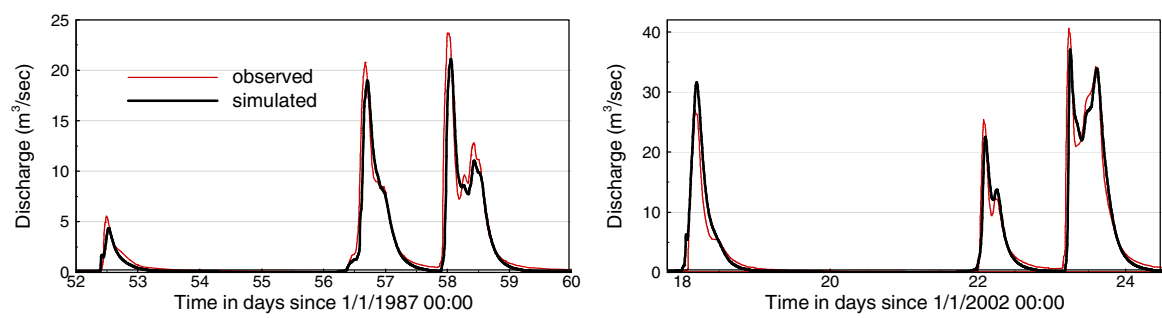

Fig. 2 GSSHA calibration (left panel) and validation (right panel). Simulations are driven by rainfall input from all rain gauges in the watershed.

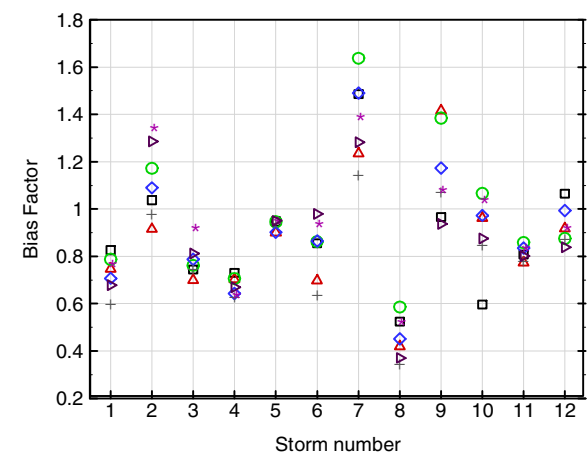

Fig. 3 Storm overall bias associated with the MPE radar data. Different symbols for each storm correspond to different pixels over the watershed.
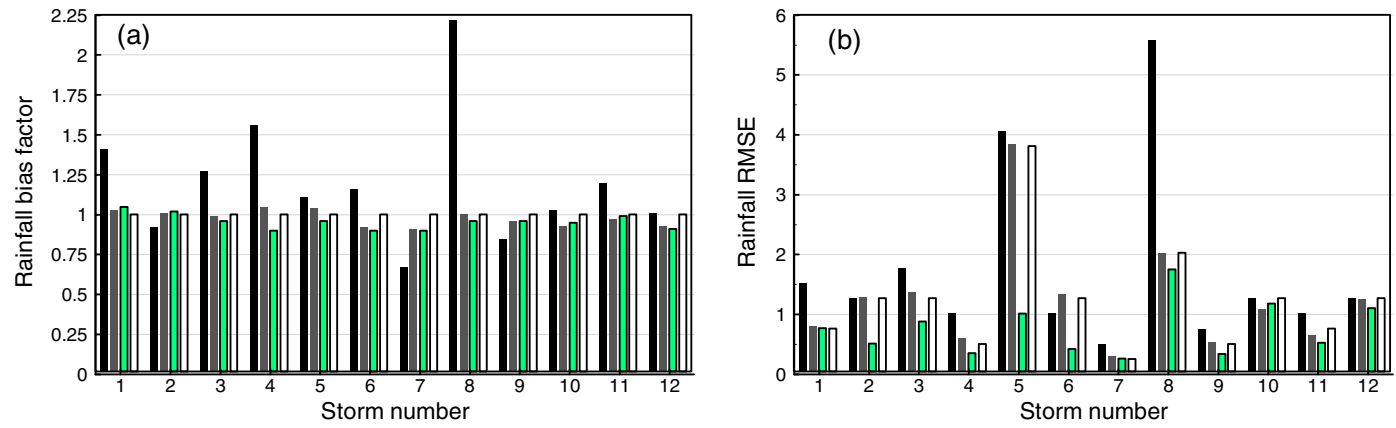

Fig. 4 Rainfall bias (a) and RMSE (b) for different bias correction methods. For each storm, column bars ordered from left to right represent the following bias adjustment methods: no adjustment, spatially-uniform unconditional, spatially-uniform conditional, and spatially-dependent unconditional (see text for more explanation). 

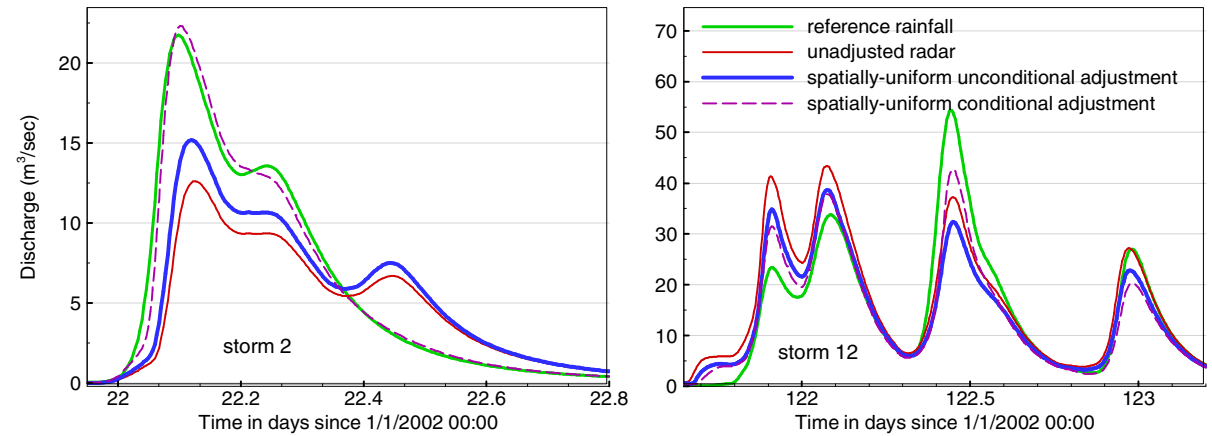

Fig. 5 Simulated runoff hydrographs using different bias adjustment methods for two example storms.
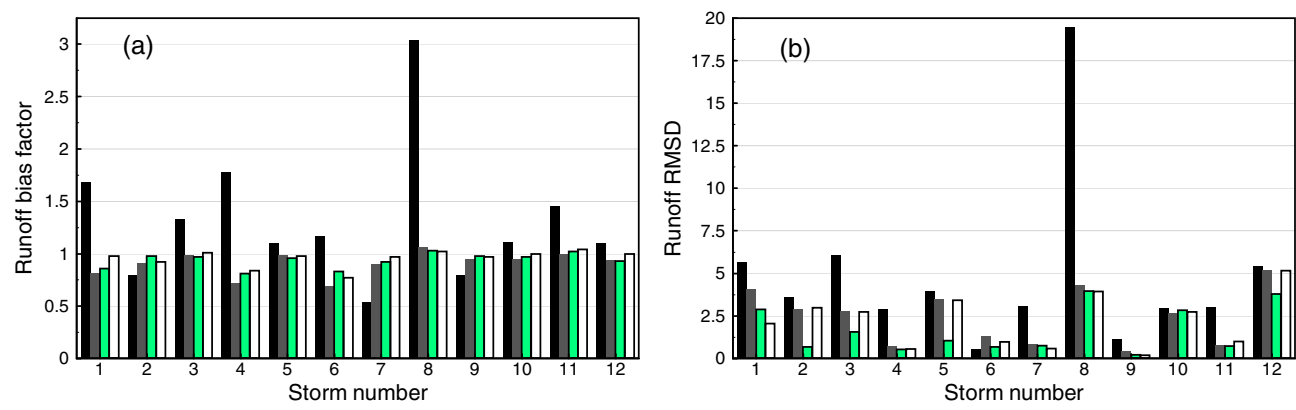

Fig. 6 Statistical assessment of effect of radar bias on runoff prediction: (a) runoff bias, (b) runoff RMSE. Column bars are the same as those in Figure 4.

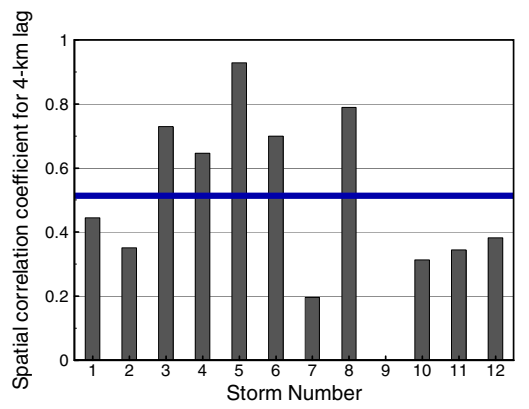

Fig. 7 Spatial correlations of the random component of the radar error at one lag $(4 \mathrm{~km})$.

Correlations are calculated between errors in three pairs of radar pixels combined together. The solid line is the correlation value after combining all storms. 

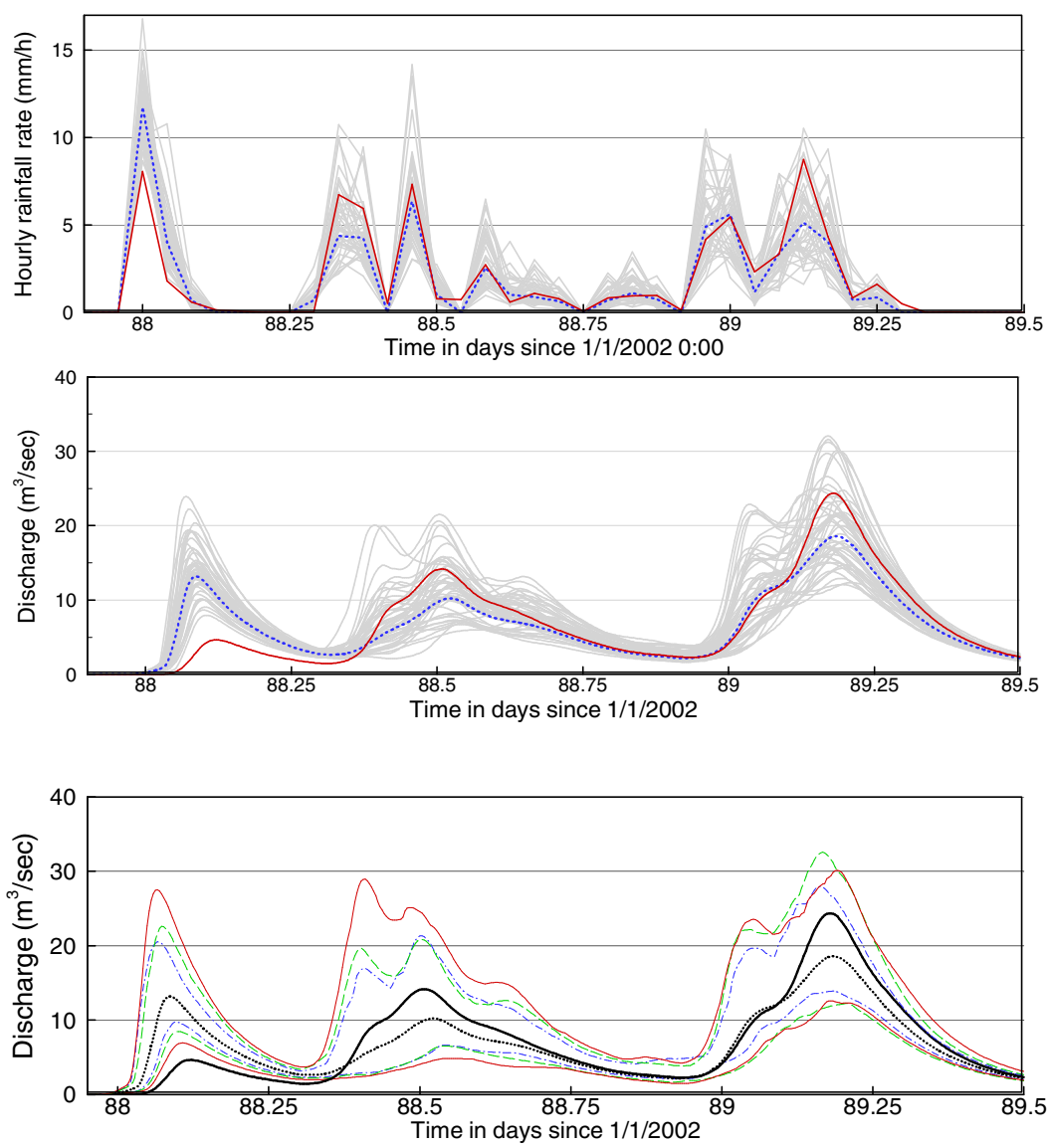

Fig. 8 Top two panels: 50 realizations of probable surface rainfall and their corresponding hydrographs generated using a value of 0.6 for the radar error spatial correlation. Also shown are reference rainfall and hydrograph (red lines) and the biasadjusted MPE radar data and hydrograph (blue lines). Lower panel shows $97.5 \%$ and $2.5 \%$ percentiles of hydrograph ensembles using spatial correlation values of: 0 (blue line), 0.6 (green line), and 0.95 (red line). The black line and black dotted lines represent runoff obtained using the reference surface rainfall and the bias-adjusted MPE data, respectively. 